\title{
Design and Implementation of Control System in a Satellite Voice Communication Terminal
}

\author{
Lirong Zhang ${ }^{1, \mathrm{a}^{*}}$ and Zhengqun $\mathrm{Hu}^{2, \mathrm{~b}}$ \\ ${ }^{1,2}$ National Astronomical Observatories, Chinese Academy of Sciences, Beijing, China \\ a Irzhang@nao.cas.cn, ${ }^{b}$ huzq@nao.cas.cn
}

Keywords: Satellite Communication; Control System; Voice-coder; Software

\begin{abstract}
A control system in satellite communication terminal of low information rate is designed via program based on ATmega128 system controller, which has realized the function of operation control in the satellite communication terminal of low information rate. The synchronization signal of clock is simulated via Mcu I/O interface to voice-coder. Mcu controls the input and output of analog signal and digital signal of voice-coder and acquires and transmits the digital signal from voice-coder. Serial communication mode is adopted between the satellite communication baseband and Mcu and the information exchange is carried out according to the system communication protocol.
\end{abstract}

\section{Introduction}

Satellite communication has some advantages such as wide coverage, high safety, high reliability, etc., which can be used as a strong supplement for ground mobile communication. Especially the establishment and development of satellite voice communication has important practical value and social significance for disaster relief and some field $[1,2]$. To improve the utilization of satellite bandwidth is one of the main directions of the development of satellite communication. Low information rate satellite voice communication can reduce the use of satellite frequency bandwidth and improve the user capacity of satellite communication voice communication system [3, 4]. A low information rate satellite voice communication system platform is constructed based on this idea. According to the operation characteristics of satellite voice communication, we design and achieve a control system in satellite communication terminal, which realizes information exchange between voice-coder and satellite communication baseband and satellite communication function of operation control.

\section{Hardware Design}

ATmega128 MCU is a 8-bit AVR microcontroller with high performance and low power [5], which is comprised by advanced RISC architecture, has powerful instruction system, integrated with large RAM of non-volatile program, data and working memory, a SPI interface, two USART interface programming, a variety of power saving modes controlled by software. It can be facilitate to achieve functions such controlling and data transmitting $[6,7]$.

A satellite voice communication terminal of low information rate is composed of ATmega128 microcontroller, voice-coder, LCD, keyboard and other circuits, which is connected to transceiver baseband through an asynchronous serial communication interface. The systematic block diagram is shown in Fig.1. 


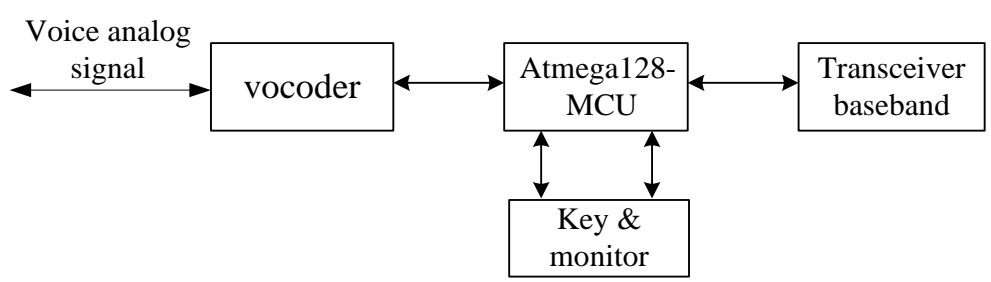

Figure 1. Systematic block diagram

Voice-coder. The hardware interface of voice-coder is defined as shown in Table 1.

Table 1 Hardware interface definition

\begin{tabular}{|c|c|c|c|}
\hline Number & Characteristics & Control Function Description & Signal Direction \\
\hline 1 & Voice_Out & Analog voice signal out & $\leftarrow$ \\
\hline 2 & Voice_In & Analog voice signal in & $\rightarrow$ \\
\hline 3 & PTT & PTT request, active at low level & $\rightarrow$ \\
\hline 4 & V_Rst & Voice-coder reset signal, active at low level & $\rightarrow$ \\
\hline 5 & \multirow[t]{2}{*}{ GND } & \multirow{2}{*}{$\begin{array}{l}\text { GND, not distinguish between analog and digital } \\
\text { ground }\end{array}$} & \\
\hline 6 & & & \\
\hline 7 & \multirow[t]{2}{*}{ VCC } & \multirow{2}{*}{$\begin{array}{l}\text { Power }(+5 \mathrm{~V}) \text {, not distinguish between analog and } \\
\text { digital power }\end{array}$} & \\
\hline 8 & & & \\
\hline 9 & RTS & Send request, active at low level & $\leftarrow$ \\
\hline 10 & TXD & Digital signal output (for voice-coder ) & $\leftarrow$ \\
\hline 11 & RXD & Digital signal input (for voice-coder ) & $\rightarrow$ \\
\hline 12 & \multirow[t]{2}{*}{ TXC } & \multirow[t]{2}{*}{ Synchronous clock signal } & \multirow[t]{2}{*}{$\rightarrow$} \\
\hline 13 & & & \\
\hline 14 & $\mathrm{CD}$ & Carrier monitoring signal, active at low level & $\rightarrow$ \\
\hline
\end{tabular}

Table 1 shows the interface definition and control function of the voice-coder and points out each pin direction (input or an output) for voice-coder. PTT is an input signal from controller to voice-coder. When it is pressed the signal is set low level by controller and the analog voice begin transmitting [8]. When RTS is set low level, the output bit stream is effective. CD is a input signal from controller to voice-coder, when it is set low level, the bits data inputting voice-coder is effective. TXD and RXD is coded bit stream with synchronization bits. The voice-coder is coded by fixed frame length. The bit stream has strict frame format. The communication channel is regarded to be transparent for voice-coder, so there is not strict frame format requirement to communication channel for the coder bit stream [9].

Interface between MCU and Voice-coder. ATmega128 control unit simulates synchronous clock signal of voice-coder transmitted via I/O [10], controls voice-coder analog signal and digital signal input and output and acquires digital signal from voice-coder and transmits digital signal to voice-coder.

The interface connected circuit between MCU and voice-coder is shown in Fig. 2. 


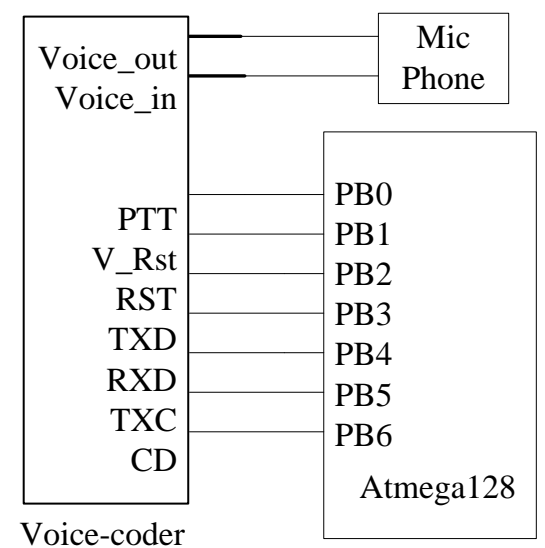

Figure 2. Hardware connected interface between MCU and voice-coder

Human-machine Interaction. Human-machine interactive part mainly includes keyboard, LCD display screen, buzzer and the indicator light. LCD is used type 12232-1with Chinese characters LCD module.

Keyboard is designed by $4 * 4$ matrix keyboard, which is defined as shown in Fig. 3.

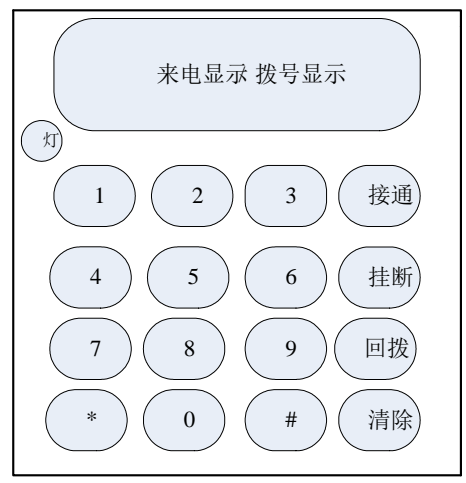

Figure 3. Terminal appearance

\section{Software Design}

Modular design is used in the systematic software. Systematic software consists of two parts: the main program and interrupt service program, they invoke other subroutine program in the implementation process. The main program includes initialization part and main loop part. The initialization part firstly initializes MCU internal resource and then initializes the peripheral important parts. MCU usually work in power saving mode, when the interrupt service subroutine wakes up MCU, corresponding subroutine will be invoked to deal with the need for immediate processing, such as the keyboard scanning, LCD display, asynchronous serial communication, etc..

Subroutine includes keyboard scanning subroutine, LCD display subroutine, serial communication subroutine, indicator light display subroutine, trigger buzzer subroutine and voice-coder communication subroutine, etc. The main program flow chart is shown in Fig. 4. Fig. 5 shows the processing flow of serial port receive interrupt. 


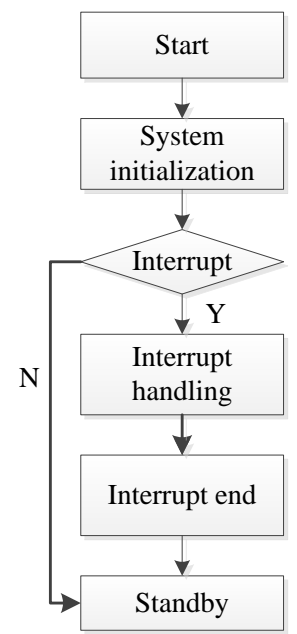

Figure 4. Main program flow

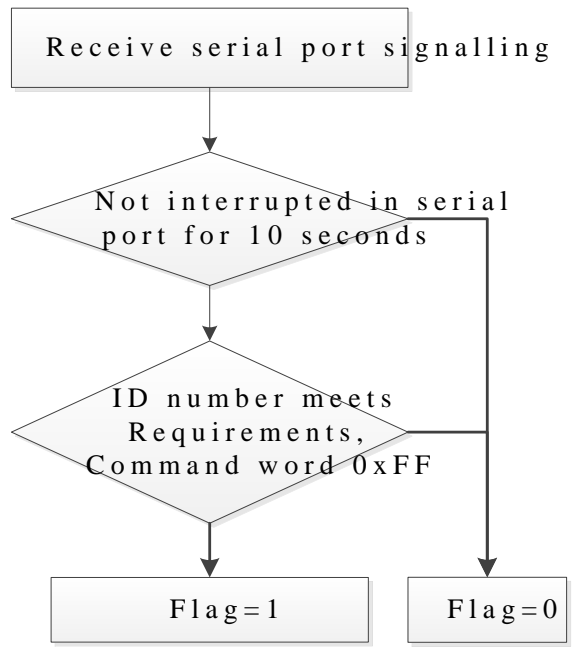

Figure 5. Processing flow of serial port receive interrupt

Interrupts service program include: keyboard key interrupt and serial communication interrupt, the entire communication process is completed in interrupt process. Fig. 6 shows the processing flow of voice telephone module.

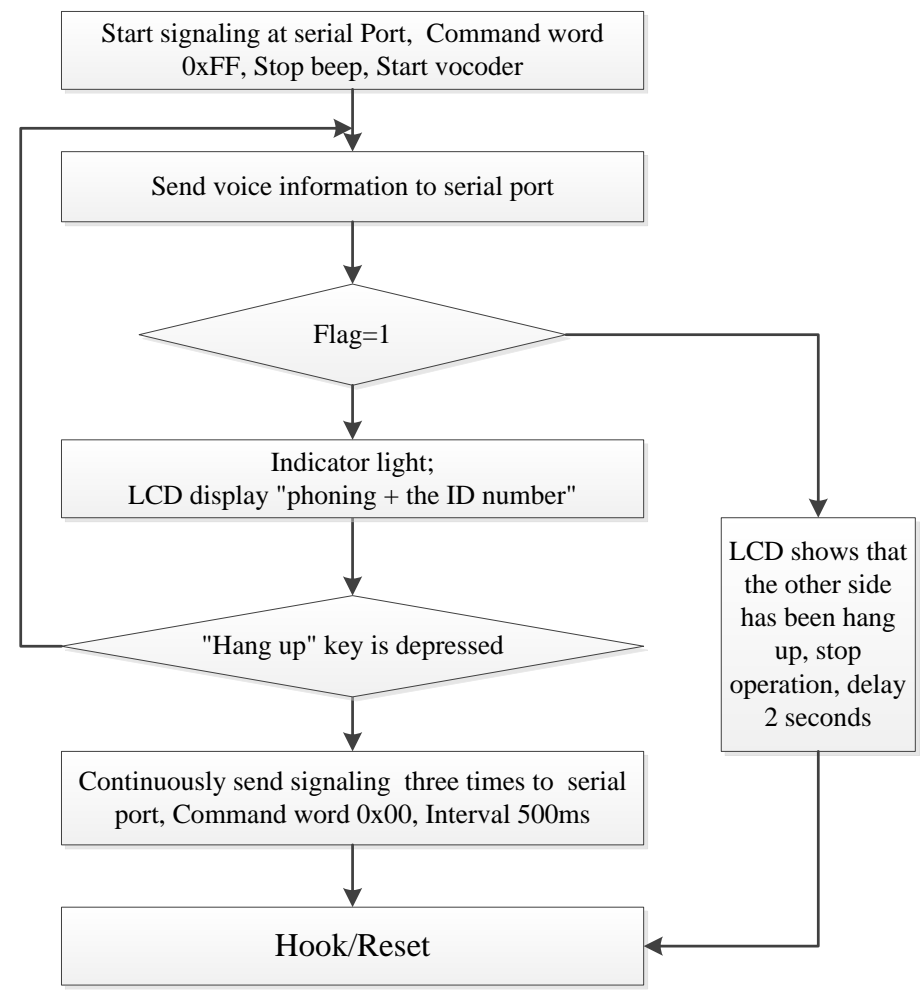

Figure 6. Processing flow of voice telephone module

\section{Conclusions}

In this paper, we put forward the idea that the low information rate voice-coder can be connected with the satellite communication system to reduce the bandwidth of satellite communication channel and improve the user capacity of satellite communication system. Based on the program of ATmega128 Mcu, a satellite voice terminal control system based on low information rate is 
designed and implemented, which includes hardware design and software design. The control system has functions of human-machine interaction, information exchange, assembly frame and so on, and set aside the expansion port which can add more functions. It will have a certain reference value in relevant technical field of control system.

\section{Acknowledgements}

The work was supported by the National Natural Science Foundation of China (11573041, 11473045, 61271284); the Young Researcher Grant of National Astronomical Observatories 2015, Chinese Academy of Sciences (Grant No.O835032002); the Key Research Program of the Chinese Academy of Sciences (KGFZD-125-14-005-2); Development Program (863 program) Project of China (Grant No.2012AA120800); the Pilot Program for New and Interdisciplinary Subjects of Chinese Academy of Sciences (KJCX2-EW-J01) and the Knowledge Innovation Program of Chinese Academy of Sciences (KGCX2-EW-4071).

\section{References}

[1] X. K. Sun: Microwave and satellite communications (Posts and Telecom Press, China 2004).

[2] B. Y. Wang: Modern satellite communication systems (Publishing House of Electronic Industry, China 2004).

[3] Y. F. Zhuang: Application of voice control technology in ship navigation system (MS., Dalian Maritime University, China 2013), p.39.

[4] S. H. Qie and J. Yang: Radio Communications Technology, Vol. 39 (2013) No.3, p.93.

[5] Z. F. Jin: C language design and Practice (Beijing University of Aeronautics and Astronautics Press, China 2008).

[6] C. Ma: High-end 8-bit microcontroller ATmega128 development and application of the principles and guidelines (Beijing Aerospace University Press, China 2004).

[7] W. Sheng: AVR microcontroller C language development guide (Tsinghua Press, China 2003).

[8] Z. Ji: Research on Low Bit Rate Speech Coding Algorithm (Ph.D., Tsinghua University, China 2011), p.26.

[9] S. B. Li, Y. F. Huang and J. C. Lu: Chinese Journal of Computers, Vol.36(2013)No.6, p.1168.

[10]X. Z. He: Application study of voice control (MS., National University of Defense Technology, China 2002), p.56. 